\title{
A Novel Approach to PID Controller Design for Improvement of Transient Stability and Voltage Regulation of Nonlinear Power System
}

\author{
Rekha Chaudhary ${ }^{1}$, Arun Kumar Singh ${ }^{1}$, Saligram Agrawal ${ }^{2}$ \\ ${ }^{1}$ Electrical Engg. Deptt., NIT Jamshedpur, India \\ ${ }^{2}$ RVSCET, Jamshedpur, India
}

\begin{tabular}{l} 
Article Info \\
\hline Article history: \\
Received Apr 12, 2016 \\
Revised Jul 13, 2016 \\
Accepted Jul 30, 2016 \\
\hline Keyword: \\
Direct feedback linearization \\
Particle swarm optimization \\
PID controller \\
Stability \\
State feedback
\end{tabular}

\section{Corresponding Author:}

Rekha Chaudhary,

Departement of Electrical and Computer Engineering,

N I T Jamshedpur, India.

Email: rchy72@gmail.com

\begin{abstract}
In this paper, a novel design method for determining the optimal PID controller parameters for non-linear power system using the particle swarm optimization (PSO) algorithm is presented. The direct feedback linearization (DFL) technique is used to linearize the nonlinear system for computing the PID (DFL-PID) controller parameters. By taking an example of single machine infinite bus (SMIB) power system it has been shown that PSO based PID controller stabilizes the system and restores the pre-fault system performance after fault is cleared and line is restored. The performance of this controlled system is compared with the performance of DFL-state feedback controlled power system. It has been shown that the performance of DFL-PID controlled system is superior as compared to DFL-state feedback controlled system. For simulation MATLAB 7 software is used.
\end{abstract}

Copyright (C) 2016 Institute of Advanced Engineering and Science. All rights reserved.

\section{INTRODUCTION}

During the past decades, the nonlinear control techniques have made great advancement. Electrical power system is an example of nonlinear system [1] whose control has attracted a lot of researchers. The major problem of electrical power system is to maintain transient stability and voltage regulation following the occurrence of sudden disturbance such as fault. The excitation system of the generator controls the terminal voltage and maintains it at constant pre-disturbance operating point [2]. To achieve the predisturbance parameters of the system, it is equipped with automatic voltage regulator (AVR) to sustain voltage variation and power system stabilizer (PSS) to provide oscillation damping [3]. The conventional AVR/PSS are designed according to the small perturbation linearized model [4]-[5]. This method suffers from the limitation that it is valid for small parametric change and hence not effective to large disturbances. In case of large disturbance, the load angle changes which may result in oscillation and the system settles at new operating point or the load angle oscillation may be increasing continuously with time and finally the system loses synchronism. Nonlinear excitation control of power generation equipment is reported [6]-[7] in which improved methodology for power system damping controller and PSS design for interconnected power system has been considered. Co-ordinated control for transient stability enhancement has been reported in recent years [8].

PID controller is the one which is widely used in the industry because of its simple structure and robust performance under wide range variation of operating conditions. The conventional PI and PID controllers are inefficient and slow in handling system non-linearity [9]. Generally the gains of the controller are tuned either by (i) manual (ii) Ziegler Nichol's method and (iii) software method. Systems that require a 
very fast rise time and /or zero overshoot cannot be tuned with Ziegler Nichol's method. For these reasons, it is desirable to add new features to controller to improve the controller performance. Artificial intelligence (AI) techniques such as neural network, fuzzy system and neural-fuzzy have been widely applied for proper tuning of PID controller [10]-[12]. Recently design of intelligent PID controller for AVR system has been carried out [13].

In recent years a number of stochastic optimization methods has been developed, among these PSO is one of the powerful method for solving optimization problem which is used here. It was developed through simulation of a simplified social system and has been found to be robust in solving continuous nonlinear optimization problems [14]-[18]. The PSO technique can generate a high quality solution with shorter calculation time and stable convergence characteristic than other stochastic methods [14]-[16]. Keeping in view of above facts, it is proposed to implement PSO to find the PID controller parameters for power system undergoing large disturbance / fault.

\section{PARTICLE SWARM OPTIMIZATION}

PSO is a population based stochastic approach for solving continuous and discrete optimization problem. In this paper, the PSO method is used for controller design of power system. Hence a brief review of the method is presented here. The PSO method was first introduced by Kennedy and Eberhart in 1995 [14]. It is a population based evolutionary heuristic optimization technique developed on the basis of social behaviour of birds flocking in search of food and fish schooling. The method has been found to be robust in solving problems with nonlinearity, non-differentiability and multiple optima. The main feature of this method is that it can be easily implemented and has stable convergence characteristic with good computational efficiency [15]. A survey of the method and its application to power system problems is available in reference [16]-[17]. PSO has been used for optimum design of PID controller in AVR system [18], where parameters of controller are tuned through PSO. The most attractive feature of the PSO method is its simplicity of application and it involves only two equations (1) and (2) associated with two vectors the position $\mathrm{X}$ and velocity $\mathrm{V}$. The method is described in brief as follows:

Each particle in PSO flies in the search space with velocity V which is dynamically adjusted according to its own flying experience and its companion's flying experience. Each particle keeps track of its co-ordinate in the problem space, which is associated with the best solution it has achieved so far. The number of variables of the optimization problem is assumed as ' $\mathrm{m}$ '. For the solution of this problem a population/swarm of ' $n$ ' particles is assumed where each particle represents a feasible solution in $m$ dimensional problem space. The position $X_{i}$ and velocity $V_{i}$ of $i^{\text {th }}$ particle is represented as: $X_{i}=$ $\left[X_{i 1}, X_{i 2}, \ldots \ldots \ldots, X_{i m}\right]$ and $V_{i}=\left[V_{i 1}, V_{i 2}, \ldots \ldots \ldots, V_{i m}\right]$ respectively.

The solution of this problem is governed by the following two equations:

$$
\begin{aligned}
& V_{i}^{k+1} \quad=V_{i}^{k}+c_{1} r_{1}\left(\text { pbest }_{i}^{k}-X_{i}^{k}\right)+c_{2} r_{2}\left(\text { gbest }^{k}-X_{i}^{k}\right) \\
& X_{i}^{k+1}=X_{i}^{k}+V_{i}^{k+1}
\end{aligned}
$$

The significance of the terms used in equation (1) and (2) are: $c_{1}$ and $c_{2}$ are two positive constants generally assumed a value of $2 ; r_{1}$ and $r_{2}$ are two randomly generated numbers within a range of [0 \& 1$]$; pbest $_{i}^{k}$ is the best position of particle i; gbest ${ }^{k}$ is the best particle position based on overall swarm's experience (having optimal cost out of the complete swarm) if the $i^{t h}$ particle has the best cost then $g$ best $t^{k}=$ $\left[X_{i 1}^{k}, X_{i 2}^{k}, \ldots \ldots \ldots, X_{i m}^{k}\right] ; \mathrm{k}$ is the iteration count.

For iterative solution of the problem initial random value of the swarm position $\mathrm{X}$ is assumed keeping in view the equality and inequality constraints of the variables. Initial value of the swarm velocity $V$ is assumed at random bounded between maximum and minimum value of velocity $\left(V_{\max }, V_{\min }\right)$ of each particle. Initial value of pbest is assumed as initial value of the position $X$. The velocity and position are improved using equation (1) and (2) respectively at each iteration keeping in view of position and velocity constraints. The iterative process stops as per the stopping criterion defined which can be the maximum number of iterations assigned or other criterion.

\section{SYSTEM REPRESENTATION AND PROBLEM FORMULATION}

The problem of power system control has been considered in this section. The power system is a large complex network represented by nonlinear mathematical model. For controlling and operation of this system a simplified power system model is considered in Figure 1. In power system dynamic study, the most 
important component is the synchronous generator with its associated excitation system and its control [3]. Although the dynamic behaviour of a synchronous generator in power system is very complicated under fault condition due to nonlinearities such as the magnetic saturation, a classical simplified third order dynamic generator model is normally used for excitation control. The classical dynamical model of a SMIB power system is described below:

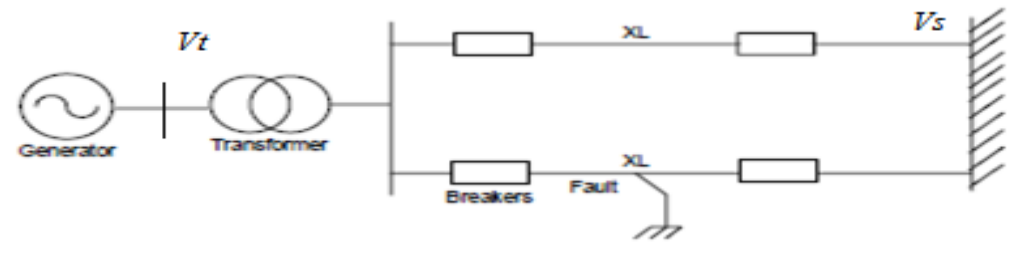

Figure 1. Schematic model of SMIB system

The power system is represented by following dynamical equations with standard assumptions and nomenclature (Appendix):

Mechanical dynamical equations:

$$
\begin{aligned}
& \Delta \dot{\delta}(t)=\omega(t) \\
& \dot{\omega}(t)=\frac{-D}{H} \omega(t)-\frac{\omega_{s}}{H} \Delta P_{e}(t)
\end{aligned}
$$

Generator Dynamical equation (Electrical):

$$
\dot{E} \dot{q}(t)=\frac{1}{T_{d o}}\left(E_{f}(t)-E_{q}(t)\right)
$$

Turbine Dynamical equation (Mechanical):

$$
\dot{P_{m}}(t)=-\frac{1}{T_{T}} P_{m}(t)+\frac{K_{T}}{T_{T}} X_{E}(t)
$$

Turbine valve control (Mechanical):

$$
\dot{X}_{E}(t)=-\frac{1}{T_{G}} X_{E}(t)+\frac{K_{G}}{T_{G}}\left[P_{c}(t)-\frac{1}{R_{G} \omega_{0}} \omega(t)\right]
$$

Electrical operational Equations:

$$
\begin{aligned}
& E_{q}(t)=\frac{x_{d s}}{x_{d s^{\prime}}} E_{q}^{\prime}(t)-\frac{x_{d}-x_{d^{\prime}}}{x_{d s^{\prime}}} V_{s} \cos \delta(t) \\
& E_{f}(t)=K_{c} u_{f}(t) \\
& P_{e}(t)=\frac{V_{s} E_{q}(t)}{x_{d s}} \sin \delta(t) \\
& I_{q}(t)=\frac{V_{s}}{x_{d s}} \sin \delta(t)=\frac{P_{e}(t)}{x_{a d^{\prime}}(t)} \\
& Q_{e}(t)=\frac{V_{s}}{x_{d s}} E_{q}(t) \cos \delta(t)-\frac{V_{s}^{2}}{x_{d s}} \\
& E_{q}(t)=x_{a d} I_{f}(t) \\
& V(t)=\frac{1}{x_{d s}} \operatorname{sqr}\left\{x_{s}^{2} E_{q}^{2}(t)+V_{s}^{2} x_{d}^{2}+2 x_{s} x_{d} x_{d s} P_{e}(t) \cot \delta(t)\right\}^{1 / 2}
\end{aligned}
$$


The power system nonlinear dynamical model is represented by equations (3) to (7). Equation (3) to (5) represents electrical dynamics and equation (6) - (7) represents mechanical dynamics. Equations (8) to (14) are the operational equations. Under small disturbance the system is controlled on the basis of linearized model (about the operating point). For large disturbance like fault on the system, if the controller is designed on the basis of linearized model the controlled system may not work satisfactorily or may lead to instability. The other problem is that the terminal voltage of the generator is also affected by large disturbance and it is an important parameter of the power system to be maintained constant for satisfactory operation.

The problem can be stated as: 'The problem is to design PID controller in the excitation loop and proportional controller in governor loop whose optimal parameters are computed with the help of PSO algorithm that will improve both the transient stability and terminal voltage simultaneously for power system undergoing large disturbance such as fault'. In this paper DFL technique is used to control the nonlinear system as discussed in the next section.

\section{DFL TECHNIQUE FOR POWER SYSTEM SOLUTION}

The stability and voltage regulation problem of power system under fault condition is stated in the previous section. The solution of this problem is considered in this section. The non-linear controller is a dynamic DFL compensator through the excitation loop to cancel the non-linearity and a robust feedback control which guarantee the asymptotic stability \& voltage regulation. To improve the performance of power system under fault condition DFL technique [19] is useful method for power system non-linear control through excitation loop. In DFL controller design the nonlinear terms of the power system are compensated by measurable output feedback [20]. In recent years design of observer based dynamic controller has emerged as a powerful tool for the system having un-measurable output variables [21]-[22].

For implementation of DFL state/output variables should be measurable. Since $E_{q}^{\prime}(t)$ in equation (5) is physically un-measurable, it is eliminated by differentiating equation for $P_{e}(t)$ (equation (10)) and using other necessary substitution one can write:

$$
\begin{aligned}
\frac{d P_{e}(t)}{d t} & =\frac{V_{s}}{x_{d s}} E_{q}(t) \cos \delta(t) \dot{\delta}(t)+\frac{V_{s}}{x_{d s}} \dot{E}_{q}(t) \sin \delta(t) \\
& =\frac{V_{s}}{x_{d s}} E_{q}(t) \cos \delta(t) \omega(t)+\frac{V_{s}}{x_{d s}} \sin \delta(t)\left[\frac{x_{d s}}{x_{d s^{\prime}}} \dot{E}_{q}^{\prime}(t)+\frac{x_{d}-x_{d}{ }^{\prime}}{x_{d s^{\prime}}} V_{s} \sin \delta(t) \omega(t)\right] \\
& =\frac{V_{s}}{x_{d s}} E_{q}(t) \cos \delta(t) \omega(t)+\frac{V_{s}}{x_{d s}} \sin \delta(t)\left[\frac{x_{d s}}{x_{d s^{\prime}}} \frac{1}{T_{d o}}\left(E_{f}(t)-E_{q}(t)\right)+\frac{x_{d}-x_{d^{\prime}}}{x_{d s^{\prime}}} V_{s} \sin \delta(t) \omega(t)\right]
\end{aligned}
$$

Equation (15), after carrying out the required substitution can be written as:

$$
\Delta P_{e}^{\cdot}(t)=-\frac{1}{T_{d o^{\prime}}} \Delta P_{e}(t)+\frac{1}{T_{d o^{\prime}}} v_{f}(t)
$$

Where

$$
v_{f}(t)=I_{q}(t)\left[K_{c} u_{f}(t)+T_{d o}{ }^{\prime}\left(x_{d}-x_{d}{ }^{\prime}\right) \frac{V_{s}}{x_{d s}} \sin \delta(t) \omega(t)\right]+T_{d o}{ }^{\prime}\left[Q_{e}(t)+\frac{V_{s}^{2}}{x_{d s}}\right] \omega(t)-P_{m}
$$

Since $P_{e}(t), I_{f}(t)$ are measurable, $I_{q}(t)$ can be computed using operational equations. $Q_{e}(t), \omega(t)$ are available by direct measurement, hence the compensating law $u_{f}$ is practically realizable which is represented by equation (18).

$$
u_{f}=\frac{1}{K_{c} I_{q}(t)}\left[v_{f}(t)-T_{d o}{ }^{\prime}\left[Q_{e}(t)+\frac{V_{s}{ }^{2}}{x_{d s}}\right] \omega(t)\right]-\frac{\left(x_{d}-x_{d}^{\prime}\right)}{K_{c}} T_{d o}{ }^{\prime} \omega(t) I_{q}(t)
$$

The model (3) to (5) is therefore linearized and it is represented by following equations (19) to (21)

$$
\begin{aligned}
& \Delta \dot{\delta}(t)=\omega(t) \\
& \dot{\omega}(t)=\frac{-D}{H} \omega(t)-\frac{\omega_{s}}{H} \Delta P_{e}(t)
\end{aligned}
$$




$$
\Delta \dot{P}_{e}(t)=-\frac{1}{T_{d o^{\prime}}} \Delta P_{e}(t)+\frac{1}{T_{d o^{\prime}}} v_{f}(t)
$$

Where $v_{f}(t)$ is the new input represented by equation (17).

For implementation of the excitation controller, $v_{f}$ is the controlled input given to the system through excitation. When composite controller (excitation and governor control) is implemented then both $v_{f}$ through excitation and $P_{c}$ through governing mechanism acting simultaneously. The detail about the controller implementation is discussed next.

\subsection{PID controller implementation}

It is assumed that the power system is operating at normal condition before the inception of fault. It is required to design a PID controller for a fault on the power system so that the controlled system is stable and attains to the pre-fault operating condition after fault is cleared. The block diagram representation for the PID controller implemented on the linearized system has been presented in Figure 2.

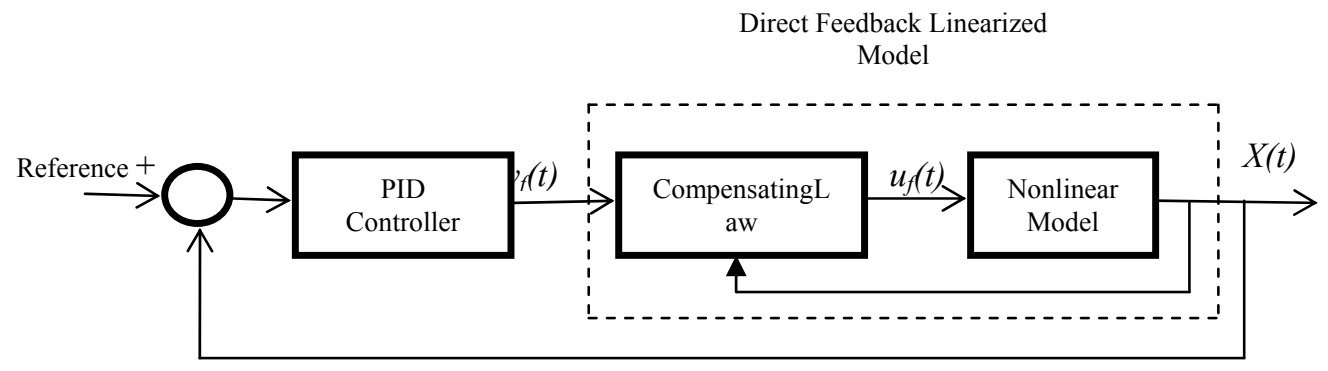

Figure 2. Power system linearized model with PID controller

In this figure $v_{f}$ is new input linearized part of the controller. $u_{f}$ is the nonlinear control input to the system composed of $v_{f}$ and the measured variable as in equation (18). $X(t)$ is the state variable of the system. The PID controller is designed to track the desired output values. The input given to the PID controller considered here is error in speed $\omega(t)$ as this quantity is easily measurable and realizable. The mathematical modelling for the PID controller to be designed is discussed below:

$$
\begin{aligned}
& e(t)=\omega(t)-\omega_{\text {reference }}=\omega(t) \\
& v_{f}=K_{P} \omega(t)+K_{I} \int \omega(t) d t+K_{D} \dot{\omega}(t)
\end{aligned}
$$

Replacing integral term $\int \omega(t) d t$ by $\left(\partial-\partial_{o}\right)$ and derivative term $\dot{\omega}(t)$ with the equation (20), equation (23) will be written as:

$$
\begin{aligned}
v_{f} & =K_{P} \omega(t)+K_{I}\left(\partial-\partial_{o}\right)+K_{D}\left[\frac{-D}{H} \omega(t)-\frac{\omega_{s}}{H}\left(P_{e}(t)-P_{m o}\right)\right] \\
& =K_{I} \partial+\left(K_{P}-K_{D} \frac{D}{H}\right) \omega(t)-K_{D} \frac{\omega_{s}}{H} P_{e}(t)+K_{D} \frac{\omega_{s}}{H} P_{m o}-K_{I} \partial_{o}
\end{aligned}
$$

Substituting the value of $v_{f}(t)$ from equation (25), $\Delta P_{e}^{\cdot}(t)$ in equation (22) one can write

$$
\begin{aligned}
& \Delta \dot{P_{e}}(t)=-\frac{1}{T_{d o^{\prime}}} \Delta P_{e}(t)+\frac{1}{T_{d o^{\prime}}} v_{f}(t) \\
& =-\frac{1}{T_{d o}{ }^{\prime}} \Delta P_{e}(t)+\frac{1}{T_{d o}{ }^{\prime}}\left[K_{I} \partial+\left(K_{P}-K_{D} \frac{D}{H}\right) \omega(t)-K_{D} \frac{\omega_{s}}{H} P_{e}(t)+K_{D} \frac{\omega_{s}}{H} P_{m o}-K_{I} \partial_{o}\right] \\
& =\frac{1}{T_{d o^{\prime}}}\left\{K_{I} \partial(t)+\left(K_{P}-K_{D} \frac{D}{H}\right) \omega(t)-\left(1+K_{D} \frac{\omega_{s}}{H}\right) P_{e}(t)+K_{D} \frac{\omega_{s}}{H} P_{m o}-K_{I} \partial_{o}\right\}
\end{aligned}
$$

The values of coefficients $K_{P}, K_{I}$ and $K_{D}$ are to be obtained with the help of PSO. Sometimes it is observed that excitation control alone stabilizes the system and regulates either load angle or terminal 
voltage. If both the load angle and terminal voltage has to be regulated then both excitation as well as governor control has to be implemented simultaneously, which is considered next.

The mechanical governing equations are written as in equation (6) and (7). Power control input, $P_{c}(t)$ is the output of governor control. It is proposed to be realized by proportional control. The relation between controller gain $K_{P C}$ and $P_{c}(t)$ is given by equation (27).

$$
P_{c}(t)=-K_{P C} e(t)
$$

The layout for PSO optimized PID controller design has been shown in Figure 3. Error $e$ is the input to the PID controller, the output of the controller is $v_{f}$ in case of excitation control and $v_{f}$ plus $P_{c}$ for both excitation and governor control. This is input to the power system to be controlled. The optimum values for $K_{P}, K_{I}, K_{D}$ and $K_{P C}$ are obtained through the PSO algorithm. The block with $\int E^{2}(t) d t$ represents the objective function as integral square error for computing the PID controller parameters by PSO in figure 3. $E(t)=Y a(t)-Y d(t)$ where $Y a(t)$ is actual output which is monitored and $Y d(t)$ is the desired value of variable (pre-fault value). There are three states: $\partial, \omega$ and $P_{e}$ so error in each state is: $E_{1}(\mathrm{t})=\partial(t)-\delta_{0}$; $E_{2}(\mathrm{t})=\omega(t)-0 ; E_{3}(\mathrm{t})=P_{e}(t)-P_{m 0}$

So the total integral square error is represented by equation (4.29)

$$
\int E^{2}(t) d t=\int E_{1}^{2}(t) d t+\int E_{2}^{2}(t) d t+\int E_{3}^{2}(t) d t
$$

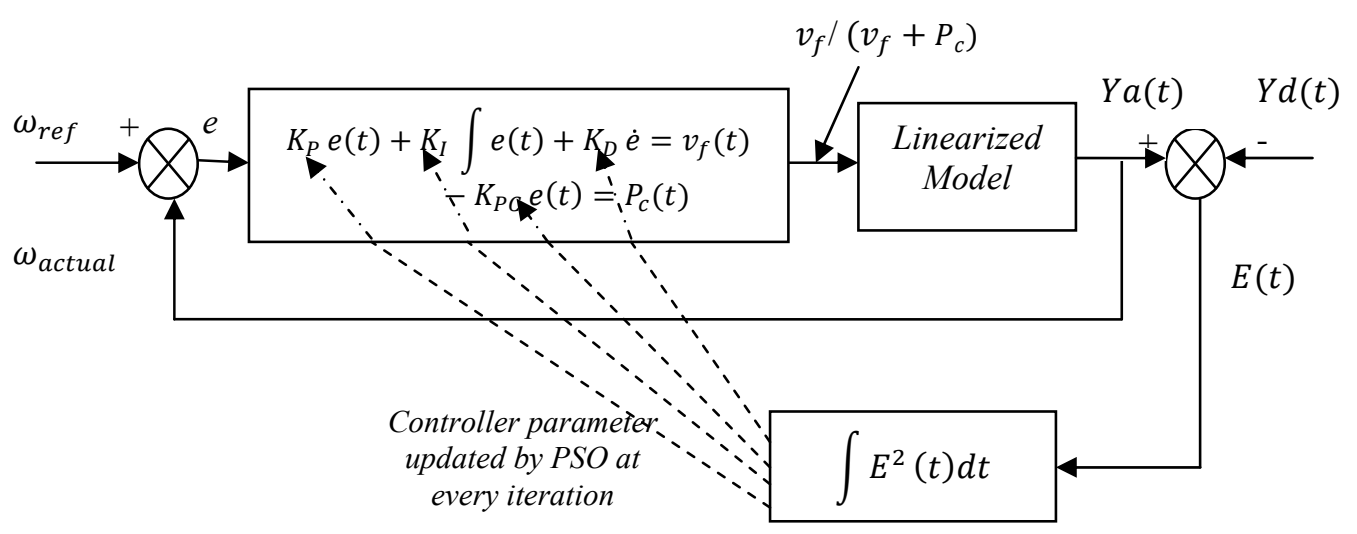

Figure 3. Block diagram for PSO based PID controller

The optimal values of controller parameters of the excitation/governor control can be obtained by applying PSO (minimizing objective function). The complete procedure is explained by taking an example of SMIB power system in next section.

\section{SYSTEM SIMULATION AND RESULTS}

For simulation SMIB system is considered as shown in Figure 1, the generator parameters have been taken from Wang et al [20] as indicated below: (saturation phenomena has not been considered). System parameters used in the simulation studies are: $x_{d}=1.863, x_{d}^{\prime}=0.257, x_{T}=0.127, T_{d o}=6.9, x_{L}=0.4853, \mathrm{H}=4$, $\mathrm{D}=5, K_{c}=1, K_{T}=1, K_{G}=1, \mathrm{R}=0.05, T_{G}=0.2, T_{T}=2.0, x_{a d}=1.712, \omega_{\mathrm{s}}=314.159$. The physical limit of excitation voltage is: $-3 \leq K_{c}$. $u_{f} \leq 6$. The normal operating point of the power system is: $\delta_{0}=47, P_{m 0}=$ $0.45 \mathrm{p} . \mathrm{u}, V_{t 0}=1.0 \mathrm{p} . \mathrm{u}$ and $\omega$ (relative speed) $=0$.

A symmetrical three-phase short circuit fault occurring on one of the transmission line is considered. Two cases of fault sequence is considered here:

- Case I: Permanent type (fault cleared)

Stage 1: the system is in a pre-fault steady-state

Stage 2: a fault occurs at time $0 \mathrm{sec}$

Stage 3: the fault is removed by opening the breakers of the faulted line at time $0.15 \mathrm{sec}$

Stage 4: the system is in a post-fault state. 
- Case II: Temporary type (fault cleared and line restored)

Stage 1: the system is in a pre-fault steady-state

Stage 2: a fault occurs at time $0 \mathrm{sec}$

Stage 3: the fault is removed by opening the breakers of the faulted line at time $0.15 \mathrm{sec}$

Stage 4: the system is in a post-fault state.

Stage 5: the system has been restored at time $1.3 \mathrm{sec}$.

For carrying out the simulation, the fault is considered at a location $\lambda=0.2$ on one of the transmission line, which signifies that the fault is at a distance of $20 \%$ of the line from the generator bus. The PID controller is designed to achieve the required system performance i.e the pre-fault value of load angle, omega and electrical power. For carrying out the design of PID controller based on excitation control, permanent type of fault (case I) has been considered and for designing the controller with excitation and governor control simultaneously, temporary type of fault (case II) is considered. For carrying out simulation, the continuous time model is discretized by first order approximation with sampling time of 0.01 sec. Continuous time system:

$\dot{X}(t)=A X(t)+B u(t)$

Discrete time system:

$\frac{X(k+1)-X(k)}{\Delta T}=A X(k)+B u(k)$

$X(k+1)=(I+\Delta T \cdot A) X(k)+\Delta T \cdot B u(k)$

$X(k+1)=A d X(k)+B d u(k)$ where $A d=(I+A . \Delta T), B d=B . \Delta T$ and $\Delta T$ is the sampling time interval.

\subsection{Simulation Results}

\subsubsection{Uncontrolled System}

The uncontrolled response of the power system after inception of is presented in Figure 4(a) and 4(b). Figure 4(a) presents the variation of power angle with time. The graph shows that the angle is increasing continuously which leads to instability. Figure 4(b) shows the variation of terminal voltage with time. The voltage profile is oscillatory and continuously decreasing with time. Thus from these two results it can be inferred that the uncontrolled system is unstable and controller is needed to stabilize the system.

In the next sub-section, PID control through excitation has been considered for stabilizing the system, PSO algorithm has been used to compute the optimum values of PID controller parameters $K_{P}, K_{I}$ and $K_{D}$. The simulation has been carried out for different types of controllers: P, PI, PD and PID controllers.

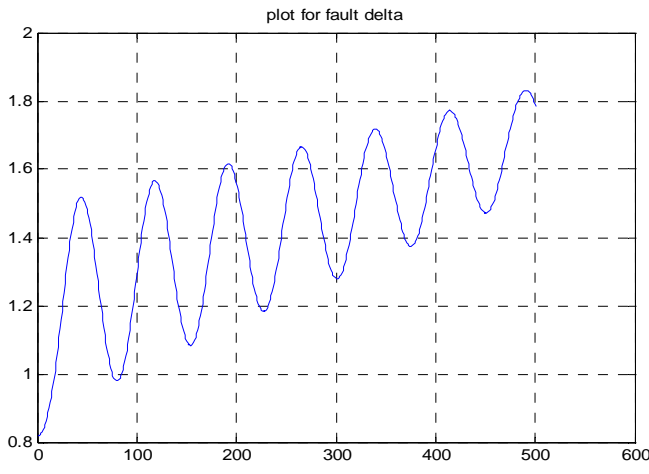

Figure 4(a). Power angle response

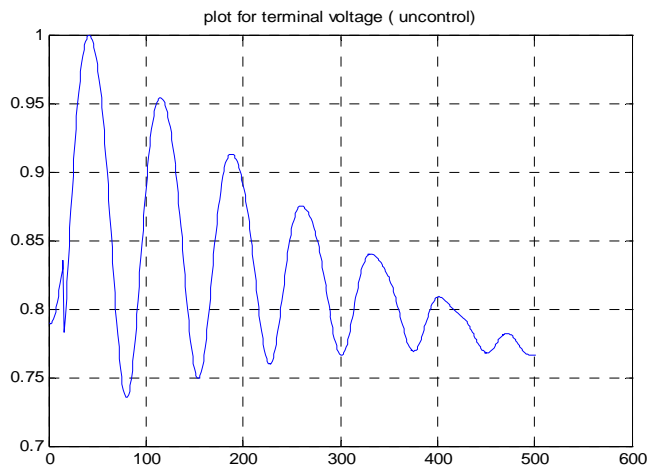

Figure 4(b). Terminal voltage response

\subsection{Excitation Control}

The three controller parameters: $K_{P}, K_{I}$ and $K_{D}$ are to be computed using PSO for excitation control (given by equation (28). The population size is assumed as four which is higher than number of variables (three) [23]. For implementation of PSO initial position (controller parameters) and velocity matrices (with velocity limit of \pm 2 ) has been assumed. For updating the particle position, dynamic PSO with weighted 
inertia has been considered. Termination of iterative process has been assumed to 300 (maximum number of iterations).

For parameter identification of PID controller, number of variables is three. Initial value of population is assumed keeping in view the parameter constraint as mentioned in table 1 and the velocity is initialized keeping in view the velocity limit. For initialization of the iteration, the initial value of pbest is considered to be initial value of population.

Table 1. Range of Controller Parameters

\begin{tabular}{ccc}
\hline Controller Parameter & Minimum Value & Maximum Value \\
\hline $\mathrm{K}_{\mathrm{P}}$ & 0 & 300 \\
$\mathrm{~K}_{\mathrm{I}}$ & 0 & 300 \\
$\mathrm{~K}_{\mathrm{D}}$ & 0 & 50 \\
$\mathrm{~K}_{\mathrm{PC}}$ & 0 & 100 \\
\hline
\end{tabular}

The proposed nonlinear control algorithm is implemented and the parameters for different types of control schemes P, PI, PD and PID are computed using PSO algorithm. The controlled response with these control schemes are simulated and are presented as follows:

\subsubsection{System response with $P$ controller}

In this case number of variable is only one $\left(K_{P}\right)$. Through PSO algorithm, value of $K_{P}$ is computed which comes out to be 3.703. The graphs for power angle (Figure 5(a)) and terminal voltage (Figure 5(b)) are plotted. These graphs show that the angle is increasing continuously with oscillatory nature and the voltage is also oscillating with magnitude decreasing. It can be inferred that with $\mathrm{P}$ control, the system cannot be stabilized and it will go out of step.

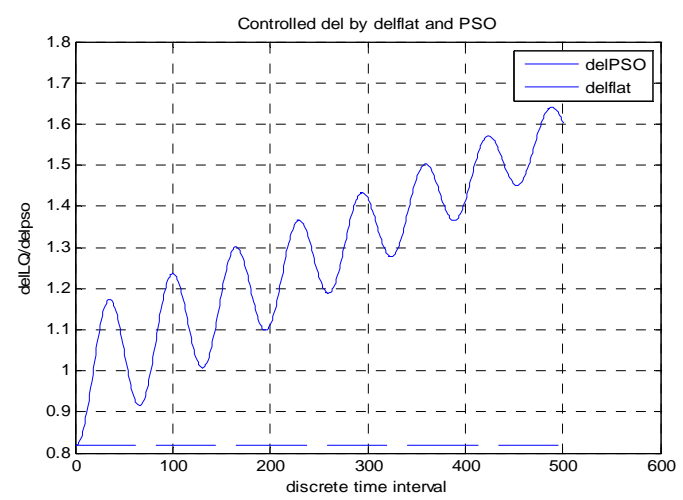

Figure 5(a). Power angle response for P control

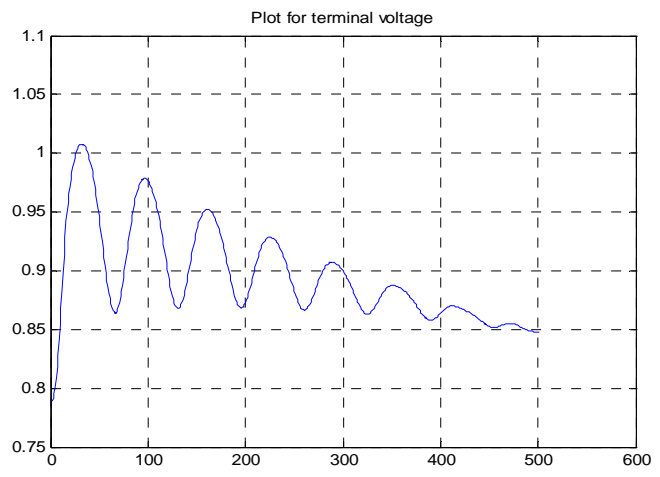

Figure 5(b). Terminal voltage response for $\mathrm{P}$ controller

\subsubsection{Response for $P I$ controller}

In this case, number of variables is: two $\left(K_{P}\right.$ and $\left.K_{I}\right)$. Through PSO algorithm, value of $K_{P}$ and $K_{I}$ are computed which comes out to be 3.703 and 0.It is observed that the controlled response is similar to the P controller as above. So the PI control is also not suitable for controlling this system.

\subsubsection{Response for PD controller}

In this case, number of variables is: two $\left(K_{P}\right.$ and $\left.K_{D}\right)$. Through PSO algorithm, value of $K_{P}$ and $K_{D}$ are computed which comes out to be 204.43 and 5.466. Figure 6(a) and 6(b) presents the results for PD controller, it is seen that the power angle is increasing continuously (slowly) with time and voltage is tending towards steady state value slowly. It is observed that the oscillation have been eliminated in this case. It is giving better result than P and PI controls. However it is to point out that as load angle is increasing continuously with time the system will finally go out of step in due course of time. Hence PD control is also not suitable. 


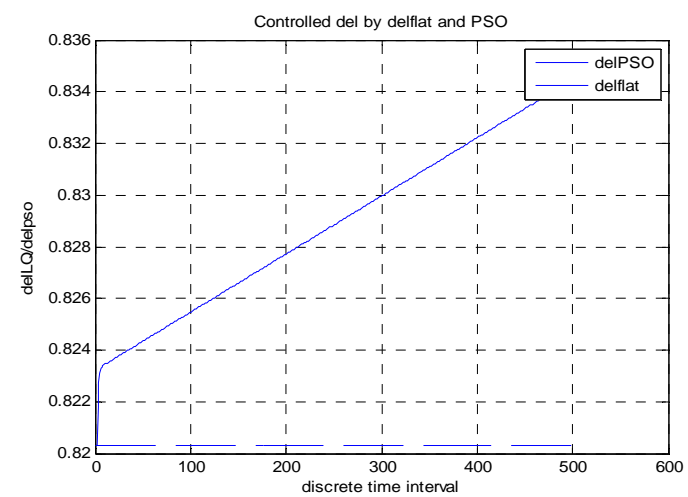

Figure 6(a). Power angle response

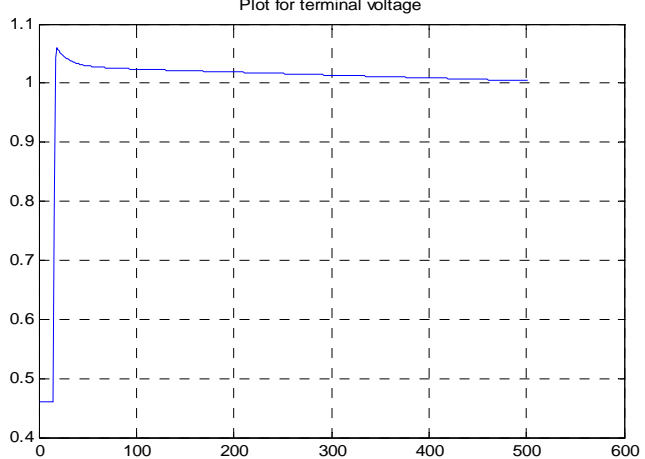

Figure 6(b). Terminal voltage response

\subsubsection{Response for PID controller}

In this case, number of variables is: three $\left(K_{P}, K_{I}\right.$ and $\left.K_{D}\right)$. Through PSO algorithm, values of these parameters are computed which comes out to be $K_{P}=169.37, K_{I}=139.77$ and $K_{D}=6.08$. With these values of controller parameters the controlled response of the system is obtained and shown in Figure 7(a) and 7(b) for power angle and terminal voltage respectively. The response shows that: (i) the controlled system is stable as the load angle and voltage both are stabilized. (ii) It is observed that the power angle has stabilized near the set point (pre-fault value) whereas the voltage settles at a value 1.04 p.u (higher than the pre-fault value 1.0 p.u). The above response shows that: among the four controllers (P, PI, PD and PID) discussed, only the PID control scheme stabilizes the power system under fault condition.

It is to point out that if voltage has also to be regulated in addition to the load angle then governor control has to be implemented in addition to excitation control. It is presented below.

\subsection{Excitation plus governor Control}

\subsubsection{Response for PID controller}

Excitation plus governor control has two inputs namely $v_{f}(t)$ and $P_{c}(t)$ (equation (25) and (28)) acting simultaneously. In designing this controller four parameters: $K_{P}, K_{I}, K_{D}$ and $K_{P C}$ (given by equation (25) and (28)) are to be computed. Here number of variables is four and the population size is assumed as five higher than number of variables [23].

The controller parameters computed through PSO are: $K_{P}=111.55, K_{I}=185.165, K_{D}=5.046$ and $K_{P C}=51.06$. The response for power angle and terminal voltage has been obtained using these controller parameters and plotted in Figure 8(a) and 8(b). It has been observed that by adding power control input $P_{c}(t)$, the performance of the system improved further. The load angle and terminal voltage tracks to the prefault condition in this case.

\subsubsection{Error comparision - excitation control and excitation plus governor control}

The error between the set point of load angle and the PID controlled system response is plotted in Figure 9(a) also the error between the terminal voltage (pre-fault value) and the terminal voltage of the controlled system is computed and plotted in Figure 9(b) for two cases excitation control only and excitation plus governor control.

Inference: For comparision the error between set-point and controlled response for third and fifth order PID controlled system has been plotted in Figure 9(a) and 9(b). It shows that in the plot of error in power angle with time (Figure 9(a)) and error in terminal voltage with time (Figure 9(a)). The error has been much reduced in case of PID controller with both excitation and governor control compare to excitation control only. In the next sub-section, DFL-state feedback controller has been discussed in brief for comparing its results with proposed PID controller. 


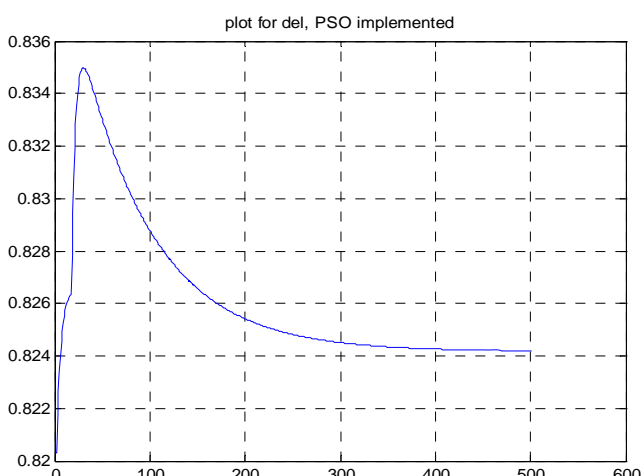

Figure 7(a). Power angle response

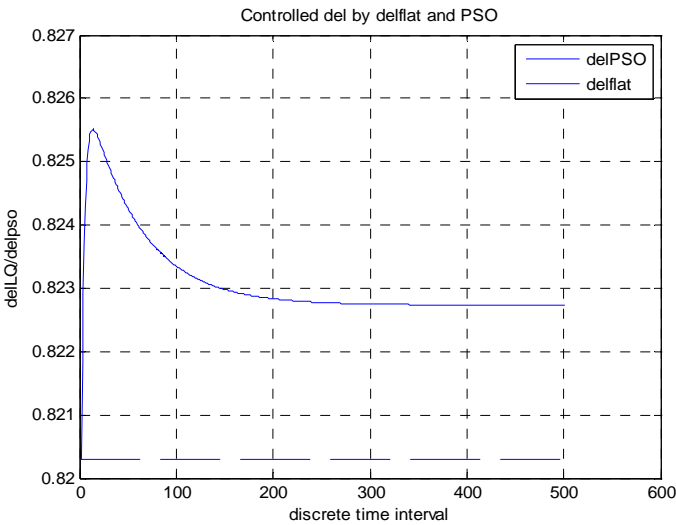

Figure 8(a). Power angle response

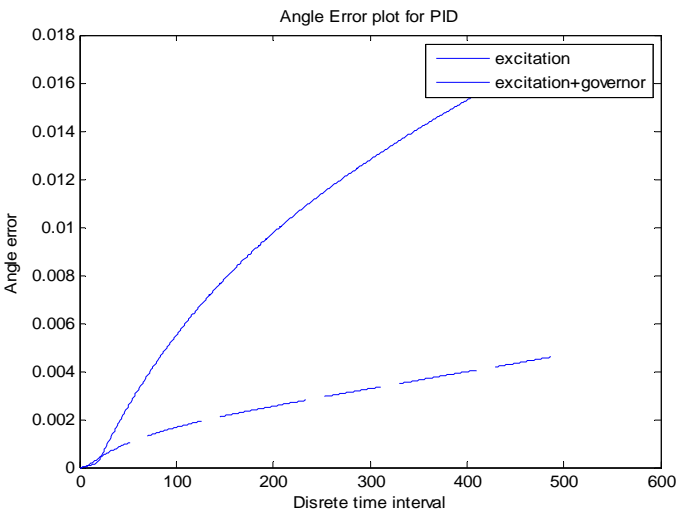

Figure 9(a). Power angle response

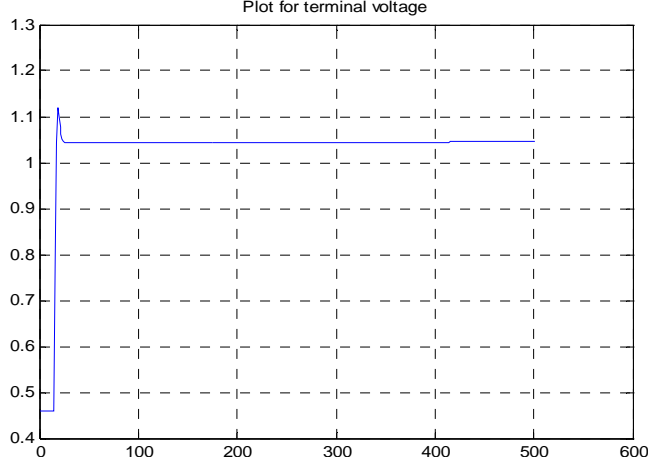

Figure 7(b). Terminal voltage response

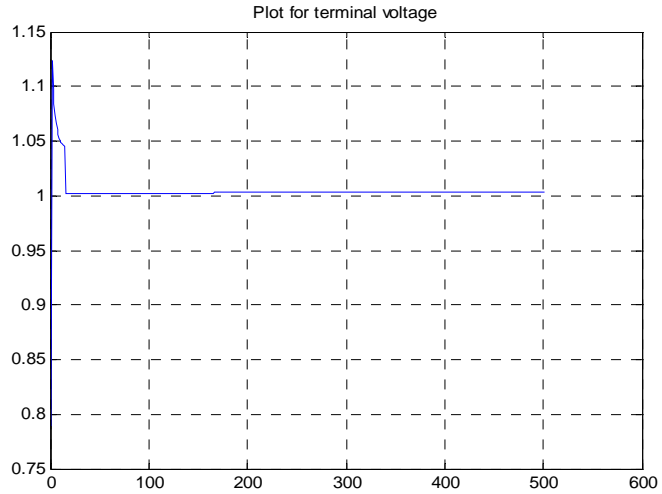

Figure 8(b). Terminal voltage response

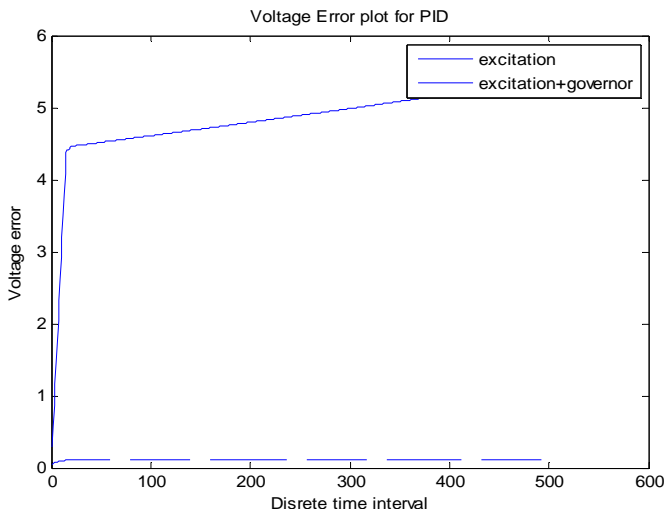

Figure 9(b). Terminal voltage response

\subsection{DFL-state feedback Controller}

\subsubsection{Response for controller}

DFL-state feedback controller design for the excitation plus governor control has been discussed here in brief. For DFL- state feedback controller design problem, the proportional error in state is used for feedback to generate the control signal as shown below:

Excitation control signal

$$
\left(v_{f}\right): v_{f}(t)=K_{1}\left(\delta(t)-\delta_{0}\right)+K_{2} \omega(t)+K_{3}\left(P_{e}(t)-P_{m 0}(t)\right)+P_{m 0}
$$


Governor control signal $\left(P_{c}\right)$ :

$$
\begin{aligned}
P_{c}(t)= & K_{11}\left(\delta(t)-\delta_{0}\right)+K_{12} \omega(t)+K_{13}\left(P_{e}(t)-P_{m 0}(t)+P_{m 0}\right. \\
& +k_{14}\left(P_{m}(t)-P_{m 0}\right)+k_{15}\left(X_{E}(t)-X_{E 0}\right)+P_{m 0}
\end{aligned}
$$

For first control input $v_{f}(t)$, the three controller parameters $K_{1}, K_{2}$ and $K_{3}$ are computed and for second input $P_{c}(t)$, five controller parameters $K_{11}, K_{12}, K_{13}, K_{14}$ and $K_{15}$ are computed using PSO. In this case the objective function is chosen as in PID control scheme for PSO implementation. The values of parameters for controlled input $v_{f}(t)$ are: 169; 152.96; -229.75, and governor control input, $P_{c}(t)$ are: 48; $17.44 ;-28.1 ; 20 ; 12.68$. With these parameter the controlled response are obtained and plotted as in Figure 10(a) and 10(b). For DFL- state feedback controller, stabilized output is produced for both power angle and terminal voltage. By adding governor control along with excitation control, the voltage has been regulated and it reaches the pre-fault value. Thus by using excitation and governor control simultaneously both deltaregulation and voltage regulation has been achieved. In the next sub-section, the error analysis has been carried out for comparing the performance of state feedback controller and PID controller.

\subsubsection{Comparision between DFL- state feedback and PID control scheme}

For error analysis, the error between the pre-fault power angle and the PID controlled angle response (excitation plus governor control) has been plotted in Figure 11(a) and the error between pre-fault terminal voltage and controlled (excitation plus governor control) terminal voltage is plotted in Figure 11(b) for both DFL-PID and DFL-state feedback.

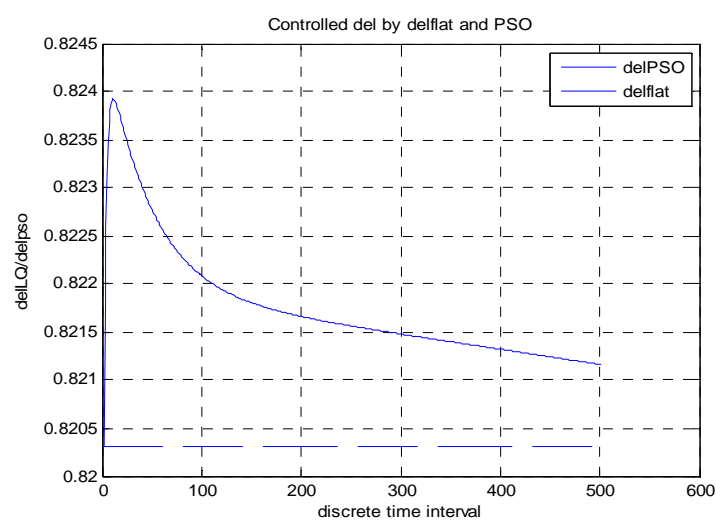

Figure 10(a). Power angle response

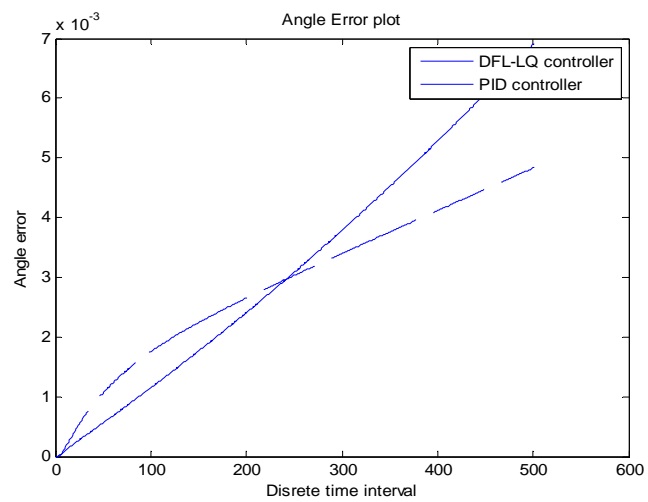

Figure 11(a). Plot for error (power angle)

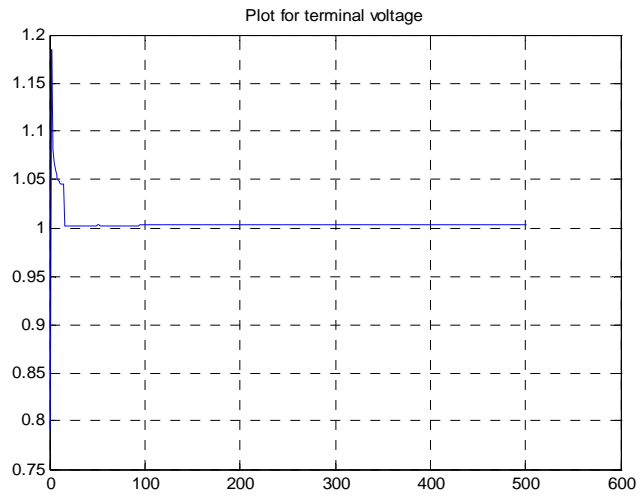

Figure 10(b). Terminal voltage response

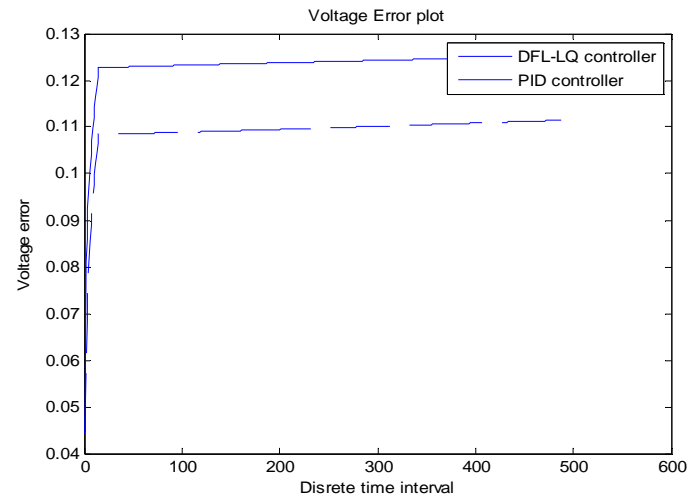

Figure 11(b). Plot for error (terminal voltage)

\subsection{Discussion}

The simulation results of uncontrolled and controlled cases of SMIB power system subjected to fault are shown by Figure 4 to Figure 11 for different control schemes. The observation is summarized as follows: 
(i) The uncontrolled system is unstable.

(ii) Excitation control for P, PI and PD does not stabilize the system (Figure 5 to Figure 6). Only PID control stabilizes the system (Figure 7). It meets the delta-regulation but voltage regulation is poor.

(iii) Response of excitation plus governor controlled system (Figure 8(a) and 8(b)) shows that the controlled system is stable and it meets both voltage regulation and delta-regulation. The error graph (Figure 9(a) and 9(b)) shows that in case of excitation and governor control, error is lower than the error graph for excitation control only.

(iv) For the sake of comparision, the response of DFL-state feedback controlled system for both the excitation and governor control input is obtained and plotted in Figure 10(a) and 10(b).

(v) The error graph for load angle shows that both PID and DFL-state feedback controller stabilizes the system. The error curve signifies that the error is lesser for DFL-PID controlled system than for the DFL-state feedback controlled system for both power angle and voltage regulation. Hence it can be inferred that the PID control scheme is viable and superior compared to DFL-state feedback controller.

\subsection{Results}

From the discussions carried out above, it is observed that among the various control schemes PID controller with both excitation and governor control is the one which stabilizes the system and restores the system pre-fault operating states in terms of both delta and voltage regulation.

\section{COMMENTS AND CONCLUSION}

In this paper PID controller design for power system undergoing fault has been presented. The controller parameters have been obtained with the help of PSO algorithm. Responses for control schemes such as P, PI, PD and PID controllers have been obtained. It is observed that only PID controlled system produces the stabilized output. Design of PID controller using only excitation control achieves the deltaregulation but voltage regulation is poor. Thus to achieve both delta-regulation and voltage regulation simultaneously, excitation and governor control both are considered simultaneously. It has been shown through error plot of load angle $\delta$ and terminal voltage $V_{t}$ using excitation control and composite control (both excitation \& governor control) (Figure 9(a) and 9(b)) that the composite control is superior in comparision to excitation control.

The responses of DFL-state feedback regulator composite control (both excitation \& governor control) has been obtained. Comparing the results of DFL-state feedback and DFL-PID, it is observed from the error plots of power angle, Figure 11(a) and terminal voltage, Figure 11(b) that the DFL-PID controller in better in terms of error minimization.

\section{ACKNOWLEDGEMENTS}

The author gratefully acknowledges the support provided by National Institute of Technology, Jamshedpur, India.

\section{Nomenclature}

\section{Appendix}

The following abbreviations are used while representing the power system model:

$\delta(t) \quad$ power angle of the generator

$\delta_{0} \quad$ power angle of the generator at the operating point (before fault)

$\omega(t) \quad$ the relative speed of the generator

$P_{m 0} \quad$ mechanical power at the operating point

$P_{m}(t) \quad$ mechanical powerinput

$P_{e}(t) \quad$ electrical power delivered by the generator

$Q_{e}(t)$ the reactive power

$\omega_{0} \quad$ synchronous machine speed; $\omega_{0}=2 \pi \mathrm{f}_{0}$

D per unit damping constant

$\mathrm{H}$ per unit inertia constant

$E_{q}^{\prime}(t) \quad$ the transient emf in the quadrature axis

$E_{f}(t) \quad$ the equivalent emf in the excitation coil

$T_{d o} \quad$ the direct axis transient short circuit time constant

$T_{d o}^{\prime} \quad$ the direct axis sub-transient short circuit time constant

$I_{f}(t) \quad$ the excitation current

$I_{q}(t) \quad$ the quadrature axis current 
$V_{t}(t) \quad$ the generator terminal voltage

$K_{c} \quad$ the gain of the excitation amplifier

$T_{T} \quad$ the time constant of turbine

$T_{G} \quad$ the time constant of speed governor

$K_{T} \quad$ the gain of the turbine

$K_{G} \quad$ the gain of the speed governor

$R_{G} \quad$ the regulation constant

$u_{f}(t)$ the input to the exciter SCR amplifier of the generator

$P_{c}(t) \quad$ the input of power control system

$X_{E}(t) \quad$ the steam valve opening

$x_{T} \quad$ reactance of the transformer

$x_{d} \quad$ direct axis reactance

$x_{d}{ }^{\prime} \quad$ direct axis transient reactance

$x_{L} \quad$ the reactance of transmission line

$x_{e d} \quad$ the mutual reactance between the excitation coil and the stator coil

$V_{S} \quad$ the infinite bus voltage

$\Delta \delta(t)=\delta(t)-\delta_{0} ; \Delta P e=P e(t)-P m ; T_{d o}^{\prime}=\frac{x_{d s^{\prime}}}{x_{d s}} T_{d o}$

$x_{d s}=x_{T}+\frac{1}{2} x_{L}+x_{d} ; x_{d s^{\prime}}=x_{T}+\frac{1}{2} x_{L}+x_{d}{ }^{\prime} ; x_{s} \quad=x_{T}+\frac{1}{2} x_{L}$ (assuming each line reactance same value)

\section{REFERENCES}

[1] A. Isidori, "Nonlinear Control Systems: An Introduction," 2nd Edn, Springer Verlag, NY, USA, 1989.

[2] Machowski J., et al., "Excitation control system for use with synchronous generators," Gen. Trans. And Distrib, IEE Proceeding, vol/issue: 145(5), pp. 537-546, 1998.

[3] Gibbard M. J., "Robust design of fixed-parameter power system stabilisers over a wide range of operating conditions," IEEE Trans. Power Syst., vol/issue: 6(2), pp. 794-800, 1991.

[4] Jabr R. A., et al., "A sequential conic programming approach for the coordinated and robust Design of power system stabilizers," IEEE Trans. Power Syst., vol/issue: 25(3), pp. 1627-1637, 2010.

[5] Jabr R. A., et al., "Robust and coordinated tuning of power system stabiliser gains using sequential linear programming," IET Gener. Transm. Distrib, vol/issue: 4(8), pp. 893-904, 2010.

[6] R. A. Ramos, et al., "An improved methodology for the design of power system damping controllers," IEEE Trans. on Power Systems, vol/issue: 20(4), pp. 1938-1945, 2005.

[7] G. Gurrala and I. Sen, "Power system stabilizers design for interconnected power systems," IEEE Trans. on Power Systems, vol/issue: 25(2), pp. 1042-1051, 2010.

[8] L. Xu, "Co-ordinated control of SVC and PSS for transient stability enhancement of multi-machine power system," TELKOMNIKA Indonesian J. of Electrical Engg., vol/issue: 11(2), pp. 1054-1062, 2013.

[9] G. J. Costa, “Tuning a PID controller," Power Transmission Engg., pp. 26-31, 2011.

[10] A. Visioli, "Tuning of PID controllers with fuzzy logic," Proc. Inst. Elect. Eng. Contr. Theory Applicat., vol/issue: $148(1)$, pp. 1-8, 2001.

[11] T. L. Seng, et al., "Tuning of a neuro-fuzzy controller by genetic algorithm," IEEE Trans. Syst., Man, Cybern. B, vol. 29, pp. 226-236, 1999.

[12] R. A. Krohling and J. P. Rey, "Design of optimal disturbance rejection PID controllers using genetic algorithm," IEEE Trans. Evol. Comput., vol. 5, pp. 78-82, 2001.

[13] K. Yavarian, et al., "Design of intelligent PID controller for AVR system using an adaptive neuro fuzzy inference system," IJECE, vol/issue: 4(5), pp. 703-718, 2014.

[14] J. Kennedy and R. Eberhart, "Particle swarm optimization," in Proc. IEEE Int. Conf. Neural Networks, Perth, Australia, vol. 4, pp.1942-1948, 1995.

[15] Y. Shi and R. Eberhart, "A modified particle swarm optimizer," in Proc. IEEE Int. Conf. Evol.Comput., Anchorage, $A K$, pp. 69-73, 1998.

[16] Y. Shi and R. C. Eberhart, "Empirical study of particle swarm optimization," in Proc. IEEE Int. Conf. Evol.Comput., Washington, DC, pp. 1945-1950, 1999.

[17] R. C. Eberhart and Y. Shi, "Comparison between genetic algorithms and particle swarm optimization," in Proc. IEEE Int. Conf. Evol. Comput., Anchorage, AK, pp. 611-616, 1998.

[18] Z. L. Gaing, "A PSO approach for optimum design of PID controller in AVR system," IEEE Trans. on energy conv., vol/issue: 19(9), 2004.

[19] L. L. Gao, et al., "A Nonlinear Control Design for Power Systems,” Auto, vol. 28, pp. 975-979, 1992.

[20] Y. Wang, et al., "Transient stability enhancement and voltage regulation of power systems," IEEE Trans. Power Syst., vol. 8, pp. 620-627, 1993.

[21] C. C. Peng and C. L. Chen, "Dynamic controller design for a class of nonlinear uncertain systems subjected to time-varying disturbance," Nonlinear Dynamics, vol. 57, pp. 411-423, 2008.

[22] M. A. Mahmud, et al., "Full order nonlinear observer-based excitation controller design for interconnected power system via exact linearization approach," Electrical Power system and Energy systems, vol. 41, 2012.

[23] S. Agrawal, et al., "Optimal power pulling for a multiple area Power system through PSO," IJEEI, vol/issue: 6(2), pp. 280-296, 2014. 


\section{BIOGRAPHIES OF AUTHORS}
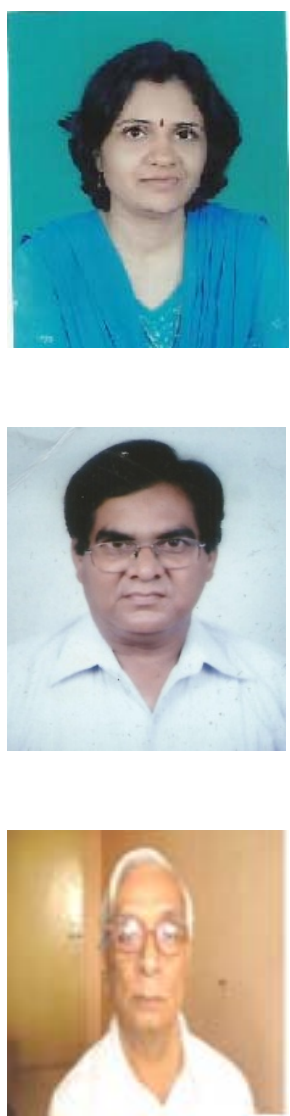

Rekha received B.Sc. Engineering degree from BIT Sindri, M.Tech in Power Systems from NIT Jamshedpur in the year 2004. She has more than 10 years of teaching experience. Currently working for her PhD at Electrical Engineering Department, NIT Jamshedpur. She has got 10 international journal papers, presented paper in 4 international conferences. Her research area includes power system operation and control, linear system theory, non-linear system theory, controller design for nonlinear system.

Arun Kumar Singh received Bachelor degree in Electrical Engineering from NIT (formerly REC) Kurukshetra, M.Tech from IIT BHU (formerly IT BHU) in the year 1985 and Ph.D from IIT Kharagpur in the year 1995. Currently he is working as Professor in Electrical Engg. Department, NIT Jamshedpur. He has teaching experience of more than 30 years. He has 21 Journal papers to his credit. His research interests include intelligent systems, linear system theory, nonlinear control, H2- H infinity control technique, robust control and power system control.

Saligram Agrawal received B.Tech and M.Tech in Electrical Engg. from NIT Jamshedpur under Ranchi University, India in year 1966 and 1978 respectively. He completed his Ph.D from IIT Delhi in 1982 in the field of power system operation and control. He is retired professor of NIT Jamshedpur and currently working as emeritus professor in private college. He has guided a number of M.Tech dissertation projects and published a number of papers in international journal. His research interests include intelligent systems, power system operation and control, heuristic methods applied in power system and control. 